\title{
Carnets
}

Revue électronique d'études françaises de l'APEF

Première Série - 2| 2010

L'équivoque

\section{Équivoque littéraire, équivoque culturelle, équivoque linguistique : «Gabriel» de George Sand et l'équivoque du genre}

\section{Giovanna di Rosario}

\section{(2) OpenEdition}

\section{Journals}

Édition électronique

URL : http://journals.openedition.org/carnets/4634

DOI : $10.4000 /$ carnets.4634

ISSN : 1646-7698

Éditeur

APEF

\section{Édition imprimée}

Date de publication : 1 janvier 2010

Pagination : 99-111

Référence électronique

Giovanna di Rosario, «Équivoque littéraire, équivoque culturelle, équivoque linguistique : «Gabriel» de George Sand et l'équivoque du genre », Carnets [En ligne], Première Série - 2 | 2010, mis en ligne le 16 juin 2018, consulté le 02 mai 2019. URL : http://journals.openedition.org/carnets/4634 ; DOI : 10.4000 /carnets.4634

\section{(c) (1) \&}

Carnets est mis à disposition selon les termes de la licence Creative Commons - Atribution - Pas d'utilisation commerciale 4.0 International. 


\title{
ÉQUIVOQUE LITTERAIRE, EQUIVOQUE CULTURELLE, EQUIVOQUE LINGUISTIQUE: «GABRIEL» DE GEORGE SAND ET L’EQUIVOQUE DU GENRE.
}

\author{
GIOVANNA DI ROSARIO \\ UOC - Universitat Oberta de Catalunya \\ Gdi_rosario@uoc.edu
}

\begin{abstract}
Résumé
Cet article vise à articuler l'équivoque mise en scène dans Gabriel de George Sand. En jouant avec l'ambigüité de la langue ce roman produit une formidable équivoque. Le titre produit une ambiguïté : une fois prononcé Gabriel n'a pas de forme féminine en français. Gabriel anticipe la pensée de Lacan : " une langue n'est rien de plus que l'intégrale des équivoques que son histoire y a laissé persister »(1973: 43). Dans Gabriel, l'équivoque se fait texte et embrasse toutes les catégories: équivoque littéraire, culturelle, linguistique. Finement, on prétend dans cet article observer les différentes formes d'équivoque qui se manifestent dans Gabriel, en mettant l'accent surtout sur la problématique du nom propre et du genre, mais aussi sur les équivoques linguistiques et culturelles.
\end{abstract}

\section{Resumo}

Este artigo visa articular o equívoco encenado em Gabriel por George Sand. Brincando com a sua língua, este romance produz um formidável equívoco: "Gabriel". O título produz também ele uma ambiguidade: uma vez pronunciado, o nome Gabriel não tem uma forma feminina em francês. Gabriel antecipa o pensamento de Lacan: " une langue n'est rien de plus que l'intégrale des équivoques que son histoire y a laissé persister » (1973: 43). Em Gabriel o equívoco faz-se texto e abarca todas as categorias: equívoco literário, cultural, linguístico. Finamente, o objectivo deste artigo consiste em observar as diferentes formas de equívoco que se manifestam em Gabriel, focalizando sobretudo a problemática do nome próprio e do género, assim como os equívocos linguísticos e culturais.

Mots-clés: Équivoque; forme; nom propre; genre.

Palavras-chave: Equívoco; forma; nome próprio; género. 
Vota pater solvit nomenque imponi avitum: Iphis avus fuerat; gavisa est nomine mater, quod commune foret nec quemquam falleret illo. Indeprensa

pia mendacia fraude letebant.

Ovidio, Metamorphoses IX, vv.708-711

[Le nom propre] prince des signifiants

Roland Barthes, L'aventure sémiologique, 1985, p. 335.

Cet article vise à articuler l'équivoque multiforme mise en scène dans Gabriel par l'extraordinaire George Sand, qui, c'est une évidence, contient déjà dans son nom une première équivoque. George Sand est le pseudonyme avec lequel Amantine Aurore Lucile Dupin (1804-1876) signait ses œuvres. Elle parlait d'elle-même sous la forme masculine ; elle portait des vêtements d'homme, elle fumait des cigares et avait de nombreux amants : elle vivait une vie d'homme dans un corps de femme qu'elle travestissait.

George Sand

Gabriel, malheureusement, est l'un de ses textes négligés (sinon oubliés) par la critique et par le public. Écrit - on connaît la proverbiale rapidité de rédaction de Sand - en quelques semaines entre mars et avril 1839, Gabriel a été publié la même année en trois épisodes par la Revue Deux Mondes: le 1er juillet : première et deuxième parties et prologue ; le 15 juillet : troisième et quatrième parties ; le 1er août : cinquième et dernière partie. En 1840 le roman a été édité en un seul volume ${ }^{1}$.

\footnotetext{
1 Pour la genèse du roman voir Glasgow, Janis (1988) préface à Sand, George. Gabriel, Paris : Édition des Femmes.
} 
Gabriel participe à la construction (romantique) de l'androgyne. Sa publication est postérieure à celle, par exemple, de Mademoiselle de Maupin (1835-36) de Gautier et d'autres ouvres de Latouche ou de Balzac pour rester dans le domaine français. Selon Annarosa Poli (1960) Gabriel recueille et condense les multiples suggestions d'où il prend origine. La question du genre étant très importante pour George Sand, le genre serait une construction sociale qu'elle a cherché à déconstruire dans plusieurs de ses romans, comme dans Indiana (1832) et Jacques (1834), mais aussi dans La Marquise (1834) qui dénonce l'éducation claustrale imposée aux jeunes-filles:

Cette éducation claustrale avait achevé d'engourdir mes facultés déjà très lentes. Je sortis du convent avec une de ces niaises innocences dont on a bien tort de nous faire un mérite et qui nuisent souvent au bonheur de toute notre vie. (p. 68)

Dans Nanon (1872), Sand brouille les genres: Nanon, tout comme Sand, refuse les contraintes du processus culturel. À noter par ailleurs le nom propre «Nanon» qui rappelle celui du page Ninon, le jeune ami(e) de Mlle de Maupin. Le nom propre révèle l'équivoque de cet androgyne: la double négation (ni-non), qui se résout en une affirmation, représente la formule neutrum/utrumque de la bissexualité/asexualité de l'androgyne. Cette formule du neutrum/utrumque, on la retrouve aussi dans Gabriel. Face à la possibilité d'une découverte publique de son vrai genre, Gabriel/le recherche l'annulation de soi-même en tant qu'individu, un individu créé par une équivoque.

Ce texte fait état de plusieurs niveaux d'équivoques: l'équivoque peut être inscrite dans le nom propre, elle peut encore être dissimulée et/ou révélée par le déguisement. Estce le vêtement qui décide du sexe ou bien le cache-t-il? Le déguisement utilisé pour révéler la nature sexuelle d'un personnage est une pratique commune à plusieurs auteurs, de Shakespeare à Th. Gautier. Dans MIle de Maupin de Gauthier (1835-36), dans As you like it de Shakespeare, il est mis en scène pour dévoiler la vraie nature de l'androgyne héroïne du roman: Madeleine d'Aubigny Maupin. Comme dans Gabriel le déguisement révèle la vraie nature de Mlle de Maupin qui, jouant le double rôle de Ganymède (homme) et Rosalinde (femme), désespère Rosette qui le croit homme et rend heureux Albert qui, grâce au déguisement, la croit maintenant femme. Mais dans Mlle de Maupin, il n'y a pas d'équivoque causée par le nom propre: deux noms sont nécessaires pour indiquer sa double nature. Au contraire dans Orlando (1928) de Virginia Woolf, le nom propre ne change pas quand Orlando devient une femme: l'équivoque du genre est déjà marquée dans son Nom Propre: OR/I/AND/o/ : or et and. Dans The Passion of New Eve (1977), c'est encore une toute autre histoire: la castration qu'Evelyn subit affecte aussi le nom propre qui se transforme en Eve, mais l'équivoque sexuelle ne se conclut pas. Finalement, pour terminer ce parcours à travers 
les romans les plus connus ayant joué sur l'équivoque du genre, il faut citer Written on the body (1992) de Jeanette Winterson, où l'absence du nom propre et du genre féminin/masculin ne permet pas de comprendre si le roman narre une histoire d'amour entre deux femmes ou entre un homme et une femme.

Dans Gabriel l'équivoque se fait texte et embrasse toutes les catégories : équivoque littéraire, culturelle, linguistique. Si « la littérature a le droit d'être équivoque » (C. Fuentes, 2005) Gabriel fait de l'équivoque sa narration aussi bien au niveau de la forme qu'au niveau du contenu.

En ce qui concerne la forme, Gebriel est un « roman dialogué », comme George Sand elle-même le définit dans sa correspondance (G. Sand, 1991). La narration procède par des dialogues : ce roman est un exemple de littérature théâtrale, il est pensé par George Sand comme une pièce théâtrale. En littérature il y a d'autres exemples d'hybridation entre le récit et la pièce théâtrale. Cependant, avec Gabriel l'aspect théâtral est prédominant. La typographie y dénonce sa structure de pièce théâtrale, bien qu'il soit techniquement impossible de la mettre en scène. Sa fruition naturelle est la lecture.

Pour ce qui est du contenu, il y a une ambiguïté totale donnée par le nom propre, par le genre sexuel de Gabriel, par le déguisement de Gabriel et par un malentendu survenant dans l'intrigue qui causera la catastrophe finale.

\section{L’histoire}

L'histoire se passe pendant une période non spécifiée de la Renaissance, dans le centre de l'Italie. La princesse, Gabriel/le, est la seule descendante de la branche la plus ancienne de la famille de Bramante. Pour des raisons dynastiques et économiques (les titres et la richesse pouvaient seulement être hérités par les enfants du sexe masculin), le prince Jules, son grand-père, lui impose, depuis l'enfance, une éducation de type masculin, à charge d'un précepteur, le révérend Chiavari. Gabriel/le vit isolée jusqu'à son dix-septième anniversaire, quand le prince décide de lui rendre visite avec l'intention de lui révéler sa vraie nature et de la persuader, pour son intérêt, de préserver une telle imposture. Mais Gabriel/le est indignée et s'échappe pour aller à la recherche de son cousin, Astolphe, descendant de la branche cadette de la famille, héritier légitime du titre, pour lui proposer de partager la propriété (prologue). Quelques jours plus tard, Gabriel, toujours déguisée en homme, trouve son cousin dans une taverne, à Florence, et elle se présente à lui. Un duel a lieu et les deux jeunes sont arrêtés et passent une nuit en prison au cours de laquelle va naître une amitié sincère. Ils deviennent des compagnons d'aventure (première partie). Après un an, pendant le carnaval, Astolphe convainc Gabriel/le de se travestir en femme. Ce déguisement produit 
un effet extraordinaire sur les amis d'Astolphe, sur la courtisane Faustina, dont Astolphe est l'amant, et sur Astolphe lui-même. Tandis qu'elle se déshabille, Gabriel/le est surprise par son cousin, qui a ainsi la confirmation de ses soupçons. Celui-ci lui déclare son amour, qui est réciproque (deuxième partie). Les deux amoureux fuient pour se marier et décident d'aller vivre chez Settimia, la mère d'Astolphe. Mais, en raison de l'hostilité de Settimia pour Gabriel/le, qui pour sa part se refuse à tous travaux domestiques, les premières difficultés surgissent (troisième partie). Gabriel/le et Astolphe décident de partir, après qu'Astolphe se soit disputé avec sa mère. Ils passent six mois à Florence, Gabriel portant pendant cette période des vêtements masculins ; et le reste de l'année, ils vivent isolés dans un palais en Calabre, où, loin des yeux indiscrets, elle est, en tous points, une femme. À cause de cette double vie, les deux amoureux commencent à se disputer : Gabriel/le ne veut pas abandonner sa double nature, et Astolphe se révèle toujours superficiel et possessif. La tragédie éclate avec l'arrivée inattendue, dans la retraite calabraise, d'un ami commun, Antonio. Astolphe enferme à clef Gabriel dans une chambre, mais Gabriel, qui n'accepte pas ce genre d'abus, s'échappe, habillée en vêtements masculins (quatrième partie). À Rome, découragée par l'attitude de son cousin et prise par des doutes au sujet de ses intentions, Gabriel prononce un vœu solennel de chasteté et de pauvreté pour obtenir du Pape la reconnaissance du total renoncement à son patrimoine en faveur du cousin Astolphe. En attendant, Astolphe, aidé par le précepteur Chiavari, va à sa recherche pour lui demander de l'épouser. Pendant la nuit de carnaval Astolphe, convaincu par une série d'équivoques de l'infidélité de Gabriel, cède aux «avances » de Faustina. Gabriel surprend la scène et réaffirme ses intentions. L'explication, donnée par le révérend Chiavari, ne produit aucun effet. Pendant la nuit, Gabriel divague à travers Rome avec l'intention de se suicider, lorsqu'elle est attaquée et assassinée par un tueur engagé par son grand-père. Le précepteur et Astolphe ne réussissent pas à empêcher la tragédie (cinquième partie).

\section{Le système onomastique et l'équivoque linguistique}

Le système onomastique ${ }^{2}$ est à la base de l'équivoque du roman : une équivoque qui se déroule tant sur le plan linguistique et sur celui de l'intrigue du roman. Gabriel porte le même nom que celui de l'archange Gabriel, peut-être doit-elle son nom à l'archange (si l'on considère l'importance de la religion à l'époque où se déroule l'action). L'archange Gabriel est, comme tous les anges, une figure sans identité sexuelle : il est asexué. L'équivoque contenue dans ce nom propre est, cependant, plus complexe. L'expédient d'un nom propre

\footnotetext{
2 Sur la théorie et la fonction du Nom Propre, voir John Searle, (1958) Proper Names, in Speech Acts, Cambridge Univesrity Press, 1966 et Marie-Noëlle Gary-Prieur, Grammaire du nom propre, Paris: PUF, 1994.
} 
homophone (sous les formes des deux genres) assure un effet remarquable d'équivocité. Au niveau phonique, le genre est au degré zéro: Gabriel-Gabrielle sont équivalents et se superposent entièrement ; au niveau oral, la double nature de «Gabriel/Gabrielle » crée l'identité de deux signifiants d'où l'équivoque ${ }^{3}$. En ce qui concerne le plan linguistique, en considérant les stratégies employées par le texte (indicateurs épicènes, reprise pronominale variable), on peut définir ce nom propre comme un nom désignant les deux genres à la fois, donc un nom équivoque.

Le composant graphique cependant, dénonce l'équivoque du signe : il n'est plus synthèse, dans sa forme écrite il est forcé de se diviser dans ses deux dimensions antithétiques. Selon Luce Irigaray « jamais une langue n'a aboli la répartition des sujets parlants en deux genres signifiant une appartenance sexuée » (Irigaray, 1987: 7). " écriture », ici, souligne immédiatement la différence de genre et les côtés de l'androgyne réapparaissent. Graphiquement le féminin est présenté comme supplément du masculin, en tant que «genre marqué » par le suffixe « elle ». D'ailleurs, c'est par ce suffixe que l'autre genre est marqué d'une manière redondante : comme morphème grammatical et comme homophone (et homographe) du pronom anaphorique « Gabrielle... « elle ». Le malentendu entre les genres ne s'arrête pas pour autant. Le rapport de dépendance entre les genres se transforme une fois de plus : le signifiant sonore de /gabrièl/ a déjà en soi (indépendamment de sa forme graphique) la marque féminine : «Gabri- (elle) ».

Le nom propre androgène ayant en soi la marque féminine suggère au lecteur la vraie nature biologique de Gabriel. Ce nom propre fait référence, simultanément, au secret et à la vérité cachée : aussi bien dans l'expression phonique que dans l'expression graphique ce nom reste double, cependant la graphie donne des indices de son équivocité. Si d'un côté la « phoné » essaie de mélanger et de confondre, de l'autre, l'écriture tend à distinguer, à diviser: la « phoné » ${ }^{4}$ et l'écriture, tôt ou tard, forcent le sujet et l'objet de l'énonciation à indiquer la sexualité, à montrer l'identité sexuelle, en adoptant le genre relatif. Cette alternance entre le genre masculin et féminin, cette équivoque linguistique est inscrite dans le nom lui-même, et elle se poursuit tout au long du texte. Ainsi le remplacement, dans la troisième partie, de «Gabriel » par «Gabrielle », se révèle comme un événement transitoire, puisque la dernière partie récupère l'indicateur onomastique initial :

Le prologue et $1^{\text {ère }}$ et $2^{\text {ème }}$ parties $\rightarrow$ Gabriel ;

$3^{\text {ème }}$ et $4^{\text {ème }}$ parties $\rightarrow$ Gabrielle ;

\footnotetext{
3 En tenant compte de la forte (même si elle n'est jamais exploitée) potentialité théâtrale de ce texte, il est facile d'imaginer l'effet de confusion que le nom propre de l'héroïne aurait produit en cas d'une réception purement orale.

4 Sur le rapport entre « phoné » et écriture voir Jaques Derrida, De la grammatologie, Paris, Minuit, 1967.
} 


\section{$5^{\text {ème }}$ partie $\rightarrow$ Gabriel}

La distinction entre les deux formes dans les différentes parties du texte et le retour final au genre masculin, cependant, n'impliquent pas pour autant la prédominance du genre masculin: la désignation reste uniformément ambiguë jusqu'à la conclusion du texte 5 . Cependant, l'écriture marque l'oscillation entre les deux côtés de l'androgyne: la désignation masculine est assez clairement connotée, insérée qu'elle est dans une classe précise des référents qui fixent les canons privés et sociaux : «l'élève » [le précepteur] (p. 68) ; «le cousin d'Astolphe » (p. 116) ; «le petit-fils [Jules] » ( $p$ 136) ; «l'héritier de la branche aînée » (p. 138) ; « le maître » [le servant Marc] (p. 221) ; « [...] Jules-Achille-Gabriel de Bramante, petit-fils, héritier présomptif et successeur légitime du très illustre et très excellent prince Jules de Bramante » (p. 200).

Cette dernière désignation dévoile l'équivoque. Le prénom, « Jules », est la marque patronymique : ce nom propre est le nom du père et du grand-père ; il est le signe de la branche la plus ancienne de la famille de Bramante. Le deuxième nom, "Achille », est l'indice de l'équivoque du genre : bien sûr il est le nom du héros grec, symbole du courage et de l'invincibilité (et, en fait, Gabriel montre du courage en désobéissant à la volonté du prince, en le contestant ouvertement; cependant, Gabrielle est petite de taille, elle ressemble à un garçon...). De plus, Achille est également un travesti, comme un lecteur sérieux pourrait le savoir :

What song the Syrens sang, or what name Achilles
assumed when he bid himself among women, although
puzzling questions, are not beyond all conjecture. (Sir Thomas Brown, E. A. Poe,
1971: 1).

Par conséquent, Achille, est un nom ambigu, androgène. Finalement, le troisième nom, «Gabriel », le nom « authentique », est celui qui par sa forme féminine, nomme réellement sa personnalité. On peut déduire une sorte d'algorithme : «Jules » masculin $\rightarrow$ «Achille » masculin et féminin $\rightarrow$ «Gabriel/le » féminin : Achille «transporte » le genre masculin au féminin. Cependant, l'équivoque masculin vs féminin se répète pendant toute I'histoire : ainsi, pendant le premier carnaval à Florence, quand Gabriel est déguisée en femme, alors qu'elle est toujours un "homme », Astolphe, qui veut tromper ses amis, l'appelle «ma fiancée » (p.115). Toutefois l'existence de «Gabrielle » en tant que femme

\footnotetext{
5 Cette division correspond à la publication en épisodes de Gabriel dans la Revue des Deux Mondes. II est probable, cependant, que cette division ait été effectuée par la revue elle-même et qu'elle ne représente pas la volonté de George Sand. Voir la correspondance de G. Sand avec Charlotte Mariani autour de la publication en revue de Gabriel: « [...] il faut qu'il en fasse seulement 2 numéros de la revue. II y a un prologue et 5 actes ou chapitres $[\ldots] \gg$ (Sand, 1991).
} 
demeure secrète et est ainsi réduite à la seule sphère privée. De cette façon, les périphrases même de "Gabrielle » montrent une capacité d'identification plus faible : «ma femme » (p. 14) ; « votre cousine » (p. 184) ; «la dame de tes pensées » (p. 197) ; « ta maîtresse » (p. 198) ; « ta Calabraise » (p. 201). Enfin dans un dialogue entre Astolphe et Antonio, Astolphe tombe dans l'équivoque du genre, en se référant à lui comme «mon cousin ou ma cousine » (p. 204). Mais, dans son rôle social, Gabriel ne peut être qu'un « homme».

\section{Gabriel vs Gabrielle : équivoque linguistique et culturelle}

II y a une disparité entre les descriptions identifiant Gabriel (en tant qu'homme) et celles qui identifient Gabrielle (en tant que femme). Le point de vue du genre change selon le personnage qui la nomme. Pour la plupart des personnages, en fait, elle est un « homme »: d'abord pour le prince Jules de Bramante, même s'il connaît sa vraie nature, Gabriel n'est acceptée qu'en tant qu'homme; le prince louera les services d'un assassin pour la tuer en raison de son instabilité " sexuelle». Elle est un homme pour les amis d'Astolphe, même s'il en est qui doutent de sa nature, comme Antonio, lequel croira que Gabrielle est un homme seulement depuis qu'elle l'a battu en duel. Elle est, au contraire, une « femme » pour Settimia, la mère d'Astolphe, mais celle-ci a également un soupçon sur sa vraie nature étant donné qu'elle est complètement étrangère aux règles imposées aux femmes par la société et qu'elle monte à cheval d'une façon qui ne convient pas à une femme (p. 132). Elle est un androgyne pour Astolphe, ainsi que pour le précepteur et pour le fidèle domestique Marc, les seuls qui soient au courant de son secret.

Par ailleurs, le lecteur suit le point de vue de Gabriel lui-même : cette analyse introspective précise l'ambiguïté et l'instabilité de son essence, elle dénonce par là même une autre équivoque. Déjà dans le prologue, le lecteur trouve quelques indices de sa vraie nature : « je ne sens pas que mon âme ait un sexe » (p.57) - il s'agit là d'un double indice, présage de la « décision finale »- ou encore «mon âme est d'une nature différente » ( $p$. 87). Dans un dialogue avec Astolphe, Gabriel lui dit : «" [...], tu m'as fait redevenir femme, mais je n'ai pas tout à fait renoncé à être homme » (p. 187) et, en fait, elle ajoute tout de suite : «II me semble toujours que je suis quelque chose de plus qu'une femme [...] ». Enfin, elle avoue : «[...] À présent, j'avoue qu'il me sera pénible de renoncer à être homme quand je veux ; car je n'ai pas été longtemps heureuse sous cet autre aspect de ma vie [...] » (p.161). La cause de cette affirmation est Astolphe, qui, par sa jalousie, a contribué à diviser le couple androgène, à dévoiler l'équivoque. Le déclassement d'Astolphe aux yeux de Gabriel lui fait réévaluer sa condition masculine: l'équilibre qui règle le couple androgène est cassé. L'éducation que le précepteur lui a transmise semble être plus forte que l'identité 
féminine, créée par la nature et structurée par Astolphe. Ainsi, en prononçant son voeu de la chasteté, de pauvreté et d'humilité, Gabriel/le sacrifie non seulement ses droits d'héritière (« sa partie masculine »), mais également sa nature : «À présent [...] je suis un garçon pour toujours » (p. 215). En fait, Gabriel/le recherche l'annulation de soi-même en tant qu'individu. Elle passe, de cette façon, de l'utrumque au neutrum. Gabriel/le veut se censurer elle-même, face à la possibilité d'une découverte publique de son vrai sexe, elle déclare :

Jamais! Je me refuse à ce dernier outrage, et, plutôt que d'en subir l'affront, je déchirerai cette poitrine, je mutilerai ce sein jusqu' à le rendre un objet d'horreur à ceux qui le verront ( $p$ 215) .

II y a une double négation : ni homme ni femme, l'être humain va vers une forme neutre, et/ou peut-être angélique. Le prologue laissait déjà pointer un indice fort pour le lecteur sur la nature «finale» de l'héroïne. Gabriel narre au précepteur un rêve étrange qu'elle a eu :

\section{GABRIEL}

J'ai rêvé que j'étais une femme. [...], je n'étais pas un habitant de cette terre. J'avais deux ailes, et je m'élevais à travers les mondes, vers je ne sais quel monde idéal. Des voix sublimes chantaient autour de moi; je ne voyais personne; mais des nuages légers [...], et j'étais une jeune fille vêtue d'une longue robe flottante et couronnée de fleurs.

\section{LE PRECEPTEUR}

Alors vous étiez un ange, et non pas une femme.

GABRIEL

J'étais une femme; car tout à coup mes ailes se sont engourdies, l'éther s'est fermé sur ma tête, comme une voûte de cristal impénétrable, et je suis tombé, tombé [...] (P. 59-60).

II y a une double équivoque : non seulement homme vs femme, mais aussi être humain vs ange. À l'intérieur du rêve une opposition forte se forme entre la femme (puis l'homme) et l'androgyne, exprimé par l'opposition « haut» vs «bas ». Quand le corps androgyne est en train de tomber, il retient la marque de la différence biologique : un lourd sein de femme. L'effet de sens produit dans l'image des ailes est lié à la partie féminine : aussi bien comme évocation du genre : « aile = elle », et comme indice du nom authentique : « j'avais deux I ». Ce rêve dévoile l'équivoque linguistique et culturelle, mais dénonce une 
autre équivoque. Ce rêve suggère la nature de Gabriel et annonce sa fin : " mon rêve me revient; il me semble que je m'envole là-haut » (p. 220). Encore une fois, il y a une opposition : ainsi, le mirage de l'altitude de l'androgyne/ange s'oppose à la chute de gravité du corps, dont le tombeau idéal est l'élément liquide, symboliquement féminin.

En simplifiant, on pourrait dire que dans la naissance et dans la mort, Gabriel/le est Gabrielle au féminin, tandis que tout au long de sa vie, la nature qui la satisfait et lui convient est la masculine:

[...] je n'ai pas tout à fait renoncé à être homme [...] II me semble toujours que je suis quelque chose de plus qu'une femme [...] je ne suis pas descendue comme femme au-dessous du rang où, comme homme, ton amitié m'avait placée [...] (p. 148-150)

Après quelques pages, en se référant à son rapport avec Astolphe, Gabriel/le affirme : « [...] À présent, j'avoue qu'il me serait pénible de renoncer à être homme quand je veux; car je n'ai pas été longtemps heureuse sous cet autre aspect de ma vie, qui est devenu notre tourment mutuel » (p.161). Elle s'est trompée en pensant pouvoir vivre avec lui comme sa femme. Ainsi c'est la dimension amoureuse qui complique l'équivoque.

Finalement, le travail de l'écriture doit permettre d'identifier et donc d'aider à résoudre l'équivoque, mais l'équivoque parcourt toute l'histoire à partir du genre, de la dimension amoureuse. Au niveau linguistique il y a une désignation mixte qui devient à la fin toujours plus brouillée : «Gabriel ... la ... elle ... il ... elle ... celle qui vous a aimé ... la ... lui ... elle» (p.123).

La mort non plus ne résout pas l'équivoque, l'androgyne ne trouve pas encore sa place: «[Astolphe en parlant à l'assassin] Misérable ! Tu l'as assassiné » (p. 123) ; « [Marc à Astolphe] Ah! Laissez-la tranquille à présent! C'est vous qui l'avez tuée » (p. 123). L'écriture ne comprend pas la nature androgène de Gabriel/le et complique l'équivoque en cherchant à la fixer sur la page, à la dévoiler. Dans la même page il y a des référents tant masculins que féminins pour Gabriel/le. Seul Astolphe comprend sa nature : «[...] toi qui n'es pas à moitié homme et à moitié femme comme tu le crois, mais un ange sous la forme humaine » (p. 151).

L'équivoque est mise en scène pendant le carnaval alors qu'Astolphe convainc Gabriel/le de se masquer avec de vêtements de femme. Astolphe est persuadé que son cousin sera fascinant déguisé en femme :

\section{ASTOLPHE}

II s'enferme vraiment comme une demoiselle. II veut que je le voie dans tout l'éclat de son costume. Je suis sûr qu'il sera charmant en fille [...] (p. 105) 
Les doutes, déjà présents chez Astolphe, augmentent lorsqu'il voit Gabriel/le habillé/e en femme. Mais il aura besoin de le/la surprendre dénudé/e (de voir son sexe biologique) pour se convaincre que son cousin est en fait une femme. C'est seulement alors que l'équivoque paraît être dévoilée.

\section{GABRIELLE}

[...] Rien! pas un vêtement, et Astolphe qui va vouloir causer avec moi en rentrant ... si je ne lui ouvre pas, j'éveillerai ses soupçons! [...] (Il prend un flambeau, ouvre une petite porte de côté et entre dans la chambre voisine. Un instant de silence, puis un cri. )

\section{ASTOLPHE}

Gabriel, tu es une femme" [...] (Tombant à genoux.) O mon Dieu, je vous remercie! ( $p$. 126)

Mais l'équivoque est inscrite en Gabriel/le, comme on l'a vu, elle est inscrite dans le nom mais aussi dans sa nature. Ainsi si l'équivoque masculin/féminin est mise en scène par le vêtement féminin porté pour le carnaval c'est encore une fois le déguisement qui anticipe la solution de l'équivoque : qui annonce la mort de Gabriel/le. Un an après, Gabriel/le se déguise pour le carnaval, mais cette fois elle porte un domino noir : "Gabriel en costume noir avec son domino rejeté sur ses épaules » (p. 205). Le domino annule le genre, il peut créer une autre équivoque. En effet autant Astolphe que Faustina le portent : "Astolphe, en domino bleu; Faustina, en domino rose» (p. 195). Mais le domino de Gabriel/le, qui a I'intention de se suicider, est noir (le noir représente symboliquement la mort et l'inconnu) ; elle cherche à s'effacer, à effacer sa nature, son identité, son équivoque.

\section{Conclusion}

L'équivoque Gabriel/le, est une équivoque qui touche la langue, le genre et la culture. Cette équivoque est insoutenable, pour Gabriel/le, pour Astolphe, qui n'accepte pas qu'elle soit un homme, pour le prince Jules qui, au contraire, ne veut pas que Gabriel sot une femme. Le lecteur souffre lui aussi de cette condition instable du texte: le lecteur voit l'équivoque quelques fois dévoilée et d'autres fois brouillée par l'écriture. L' «être du manque " n'arrive pas à trouver un équilibre et l'écriture non plus. La fonction de cohésion (anaphorique et cataphorique), typique du nom propre, n'est pas accomplie dans ce texte. Le pacte de lecture, basé sur plusieurs degrés d'équivoques, est rompu puisque tous, le prince Jules, le précepteur Chiavari, Marc, Astolphe (et le lecteur aussi) ont cherché à 
effacer l'équivoque inscrite en Gabriel/le, à lui donner une identité stable. Mais la nature de Gabriel est ambiguë, la seule solution possible sera donc la mort. 


\section{Bibliographie}

BARTHES, Roland (1973). "Analyse textuelle d'un conte d'Edgar Poe". In L'aventure sémiologique. Paris : Seuil, 1985.

Derrida, Jaques (1967). De la grammatologie. Paris : Minuit.

DURUPT, Beatrice (1993). Combien de sexes avez-vous?, "Diagraphe", 63.

FUENTES, Carlos (2005). Le Monde Diplomatique, " Le Monde diplomatique" [on-line]. Francer (actualisé le Décembre 2005). <URL: http://www.mondediplomatique.fr/2005/12/FUENTES/13007>

GARY-PRIEUR, Marie-Noëlle (1994). Grammaire du nom propre. Paris: PUF.

GLASGow, Janis (1988). Préface à SAND, George, Gabriel. Paris : Edition des Femmes.

IRIGARAY, Luce (1987). Le sexe linguistique, "Langages", 85.

LACAN, Jaques (1973). "L'étourdit". In Scilicet n4, pp.5-52.

OVIDIO, Nasone Publio (2007). Metamorphose., Milano: Mondadori.

POE, Edgar Allan (1841). “The Murders In The Rue Morgue”. London: Paperback, 2001.

PolI, Annarosa (1960). L'Italie dans la vie et l'oeuvre de George Sand. Paris: Armand Colin.

SAND, George (1991). Correspondance. Paris: Garnier (vol. IV).

SAND, George (1988). Gabriel. Paris: Edition des Femmes.

SAND, George (1986). Nouvelles. Paris : Edition des Femmes.

SeARLE, John R. (1958). Proper Names. In Speech Acts, Cambridge University Press: 1966. 